TITLE:

\title{
Electromagnetic Field Computation Using Space-Time Grid and Finite Integration Method
}

$\operatorname{AUTHOR}(S)$ :

Matsuo, Tetsuji

\section{CITATION:}

Matsuo, Tetsuji. Electromagnetic Field Computation Using Space-Time Grid and Finite Integration Method. IEEE Transactions on Magnetics 2010, 46(8): 3241-3244

\section{ISSUE DATE:}

2010-07-19

URL:

http://hdl.handle.net/2433/226608

\section{RIGHT:}

(c) 2010 IEEE. Personal use of this material is permitted. Permission from IEEE must be obtained for all other uses, in any current or future media, including reprinting/republishing this material for advertising or promotional purposes, creating new collective works, for resale or redistribution to servers or lists, or reuse of any copyrighted component of this work in other works.; この論文は出版社版でありません。引用の際には出版社版をご確認ご利用ください。; This is not the published version. Please cite only the published version. 


\title{
Electromagnetic Field Computation Using Space-Time Grid and Finite Integration Method
}

\author{
Tetsuji Matsuo, Member, IEEE,
}

\begin{abstract}
A finite integration (FI) method on a space-time grid is studied for computation of electromagnetic wave propagation, where a nonuniform time-step distribution is naturally introduced. Orthogonality between dual grids is given by the Lorentz metric to obtain a constitutive equation for electromagnetic variables. Electromagnetic field computations show that the FI method on a nonuniform space-time grid yields correct wave propagation.
\end{abstract}

Index Terms-Finite integration method, Lorenz metric, spacetime grid.

\section{INTRODUCTION}

$\mathbf{T}$ HE FINITE difference time domain (FDTD) method [1] is widely used for electromagnetic field computation because of its efficiency and accuracy. However, the conventional Yee grid requires brick-type representation of analyzed objects. The finite integration (FI) method [2], [3] can use flexible spatial grids including tetrahedral, prismatic, and pyramidal elements. Both conventional FDTD and FI methods use a uniform time-step. The FDTD method using sub-grids [4], [5] uses nonuniform time-steps, but uses only brick-type spatial grids.

In contrast, space and time are handled in a unified manner by the special theory of relativity. Although usual electromagnetic field computation requires no relativity theory, electromagnetic fields can be analyzed in space-time because Maxwell equations are unaffected by the special theory of relativity. Actually, several space-time finite element methods [6]-[8] have been proposed for eddy-current analysis.

This study examines a space-time FI method for electromagnetic wave propagation, where nonuniform time-step distribution is naturally introduced.

\section{Finite Integration Method on a Space-Time GRID}

\section{A. Electromagnetics in Space-Time}

The Euclid metric is used in this article for an analogical explanation to the conventional electromagnetics in two- or three-dimensional (2D or 3D) space.

For simplicity, permittivity $\varepsilon$ and permeability $\mu$ are assumed to be constant. A time-variable $w$ is introduced as

$$
w=c t, c=1 / \sqrt{\varepsilon \mu} \text {. }
$$

A 2D-electromagnetic field is described by (2) and (3), where $E_{x}, E_{y}$ and $B_{z}$ propagate in $(x, y, w)$ space-time.

$$
\partial \mathcal{E}_{y} / \partial x+\partial\left(-\mathcal{E}_{x}\right) / \partial y+\partial B_{z} / \partial w=0
$$

T. Matsuo is with the Department of Electrical Engineering, Kyoto University, Kyoto, 615-8510 Japan, e-mail: tmastuo@kuee.kyoto-u.ac.jp

$$
\begin{gathered}
\partial \mathcal{H}_{z} / \partial y-\partial D_{x} / \partial w=0,-\partial \mathcal{H}_{z} / \partial x+\partial\left(-D_{y}\right) / \partial w=0 \\
\mathcal{E}_{x}=E_{x} / c, \mathcal{E}_{y}=E_{y} / c, \mathcal{H}_{z}=H_{z} / c
\end{gathered}
$$

Two electromagnetic vectors are formally defined in $(x, y$, w) space-time as

$$
\boldsymbol{F}=\left(\mathcal{E}_{y},-\mathcal{E}_{x}, B_{z}\right), \boldsymbol{G}=\left(-D_{y}, D_{x}, \mathcal{H}_{z}\right)
$$

Equations (2) and (3) can be rewritten with $\boldsymbol{F}$ and $\boldsymbol{G}$ as

$$
\nabla_{2} \cdot \boldsymbol{F}=0, \nabla_{2} \times \boldsymbol{G}=0
$$

where $\nabla_{2}$ means $(\partial / \partial x, \partial / \partial y, \partial / \partial w)$.

The integral form of (6) is given as

$$
\oint_{\mathrm{S}} \boldsymbol{F} \cdot \boldsymbol{n} \mathrm{d} S=0, \oint_{\mathrm{C}} \boldsymbol{G} \cdot \boldsymbol{t} \mathrm{d} s=0
$$

where $\boldsymbol{n}$ and $\boldsymbol{t}$ respectively denote the unit normal and tangential vectors.

Equation (7) derives an FI method in space-time using electromagnetic variables defined as

$$
\begin{gathered}
e_{x}=\int \mathcal{E}_{x} \mathrm{~d} w \mathrm{~d} x, e_{y}=\int \mathcal{E}_{y} \mathrm{~d} y \mathrm{~d} w, b=\int B_{z} \mathrm{~d} x \mathrm{~d} y \\
d_{x}=\int D_{x} \mathrm{~d} y, d_{y}=\int D_{y} \mathrm{~d} x, h=\int \mathcal{H}_{z} \mathrm{~d} w
\end{gathered}
$$

The integration of $d_{x}, d_{y}$, and $h$ along the $z$-direction is assumed implicitly for unit length in (9).

When orthogonal primal and sub grids in $(x, y, w)$ spacetime are used, the FI method reduces (7) to (see Fig. 1)

$$
\begin{gathered}
-b_{i, j}^{n-1}+e_{x, i, j-1 / 2}^{n-1 / 2}-e_{y, i-1 / 2, j}^{n-1 / 2} \\
+b_{i, j}^{n}-e_{x, i, j+1 / 2}^{n-1 / 2}+e_{y, i+1 / 2, j}^{n-1 / 2}=0 \\
-d_{y, i+1 / 2, j}^{n-1 / 2}-h_{i, j}^{n}+d_{y, i+1 / 2, j}^{n+1 / 2}+h_{i+1, j}^{n}=0 \\
+d_{x, i, j+1 / 2}^{n-1 / 2}-h_{i, j}^{n}-d_{x, i, j+1 / 2}^{n+1 / 2}+h_{i, j+1}^{n}=0 .
\end{gathered}
$$

Those result in the conventional FDTD scheme.

The following electromagnetic variables are defined when a non-orthogonal primal grid and its dual grid are used.

$$
f=\int \boldsymbol{F} \cdot \boldsymbol{n} \mathrm{d} S, g=\int \boldsymbol{G} \cdot \boldsymbol{t} \mathrm{d} s
$$

Variables $f$ and $g$ are defined, respectively, on the primal and sub grids. Integration of $g$ along the $z$-direction is assumed implicitly for unit length in (13).

Let $\boldsymbol{n}_{\mathrm{p}}=\left(n_{1}, n_{2}, n_{3}\right)$ be the normal vector of a face of the primal grid. To relate $f$ to $g$, the tangential vector of the corresponding edge of the sub grid is given as $\boldsymbol{t}_{\mathrm{s}}=\left(-n_{1},-n_{2}, n_{3}\right)$, 


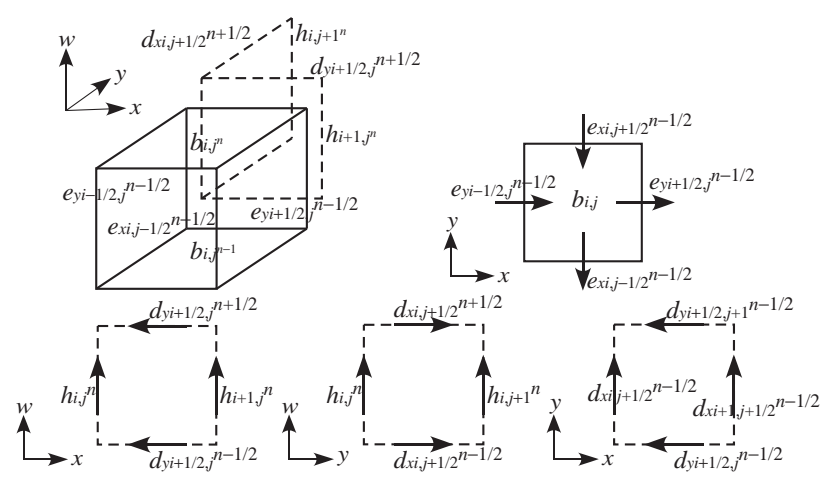

Fig. 1. FI method using an orthogonal space-time grid. The arrow directions correspond with the definition (5) of $\boldsymbol{F}$ and $\boldsymbol{G}$ and the integral forms (7). They do not correspond with the directions of $\boldsymbol{E}, \boldsymbol{D}$ and $\boldsymbol{H}$ in the space directly.

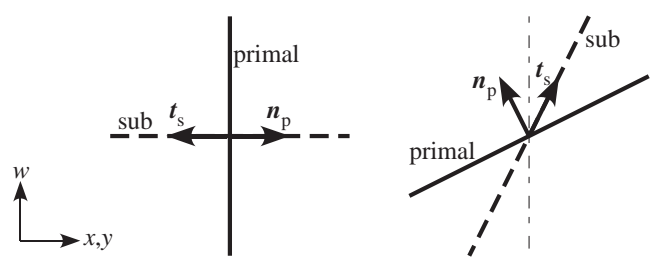

Fig. 2. Relation between the face of a primal grid and the edge of a sub grid.

as presented in Fig. 2. Thereby,

$$
\begin{aligned}
\boldsymbol{F} \cdot \boldsymbol{n}_{\mathrm{p}} & =\mathcal{E}_{y} n_{1}-\mathcal{E}_{x} n_{2}+B_{z} n_{3} \\
& =\sqrt{\mu / \varepsilon}\left(D_{y} n_{1}-D_{x} n_{2}+\mathcal{H}_{z} n_{3}\right)=Z \boldsymbol{G} \cdot \boldsymbol{t}_{\mathrm{s}}
\end{aligned}
$$

where $Z=\sqrt{\mu / \varepsilon}$. In fact, $\boldsymbol{t}_{\mathrm{s}}$ is orthogonal to the corresponding face of the primal grid by the Lorentz metric.

\section{B. Explicit Time-Marching}

For the space-time FI method to be as efficient as the conventional FDTD method, an explicit time-marching scheme is required. An explicit scheme is given as follows.

For simplicity, a scheme for 2D space-time with 1D space along the $x$-direction is derived where $\mathcal{E}_{x}=0$. Fig. 3 presents a space-time grid in which the solid and dashed lines respectively denote edges of primal and sub grids. The grid has three domains according to the time-step: (I) the domain with timestep $\Delta w$, (II) the domain with $\Delta w / 2$, and (III) the domain connecting (I) and (II). For simplicity, $\varepsilon$ and $\mu$ are assumed to be uniform and $\Delta x$ is uniformly set to unity.

In domain (I), $d_{y}$ and $e_{y}$ are updated as

$$
\begin{aligned}
& d_{y, i-1 / 2}^{n+1 / 2}=d_{y, i-1 / 2}^{n-1 / 2}-\left(h_{i}^{n}-h_{i-1}^{n}\right), \\
& e_{y, i-1 / 2}^{n+1 / 2}=(Z \Delta w) d_{y, i-1 / 2}^{n+1 / 2} \quad(i \leq I-1) .
\end{aligned}
$$

In domain (II), $d$ and $e$ are updated as

$$
\begin{aligned}
& d_{y, i-1 / 2}^{n+1 / 4}=d_{y, i-1 / 2}^{n-1 / 4}-\left(h_{i}^{n}-h_{i-1}^{n}\right), \\
& e_{y, i-1 / 2}^{n+1 / 4}=(Z \Delta w / 2) d_{y, i-1 / 2}^{n+1 / 4}(i \geq I+2) .
\end{aligned}
$$

In connecting domain (III), $d$ and $e$ are updated as

$$
\begin{aligned}
& d_{y, I+1 / 2}^{n+1 / 4}=d_{y, I+1 / 2}^{n-1 / 4}-\left(h_{I+1}^{n}-h_{I}^{n}\right), \\
& e_{y, I+1 / 2}^{n+1 / 4}=(Z \Delta w / 2) d_{y, I+1 / 2}^{n+1 / 4} /\left(1 / 2+\Delta l_{\mathrm{A}}+\Delta w^{2} / 8\right)
\end{aligned}
$$

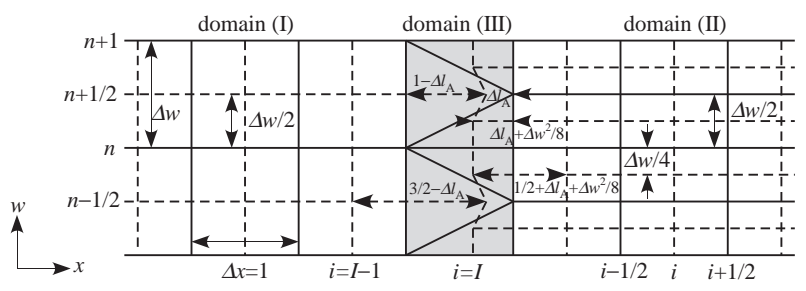

Fig. 3. Space-time grid with 1D space.


Fig. 4. Variables in the connecting domain. The arrow directions correspond with the definition (5) of $\boldsymbol{F}$ and $\boldsymbol{G}$ and the integral forms (7).

where $0<\Delta l_{\mathrm{A}}<1 / 2-\Delta w^{2} / 8$ (see Fig. 3). Then, $f^{n+1 / 4}$ and $g^{n+1 / 4}$ in domain (III) are given as (see Fig. 4(a))

$$
f^{n+1 / 4}=-e_{I+1 / 2}^{n+1 / 4}+b_{I}^{n}, g^{n+1 / 4}=(\Delta w / 4 / Z) f^{n+1 / 4} .
$$

In domain (II), $b$ and $h$ are updated as

$$
\begin{aligned}
b_{i}^{n+1 / 2} & =b_{i}^{n}-\left(e_{y, i+1 / 2}^{n+1 / 4}-e_{y, i-1 / 2}^{n+1 / 4}\right), \\
h_{i}^{n+1 / 2} & =(\Delta w / 2 / Z) b_{i}^{n+1 / 2} \quad(i \geq I+1) .
\end{aligned}
$$

Subsequently, $d_{y}$ and $e_{y}$ in domain (II) are updated as

$$
\begin{aligned}
& d_{y, i-1 / 2}^{n+3 / 4}=d_{y, i-1 / 2}^{n+1 / 4}-\left(h_{i}^{n+1 / 2}-h_{i-1}^{n+1 / 2}\right), \\
& e_{y, i-1 / 2}^{n+3 / 4}=(Z \Delta w / 2) d_{y, i-1 / 2}^{n+3 / 4}(i \geq I+2) .
\end{aligned}
$$

In the connecting domain (III), $d_{y}$ and $e_{y}$ are updated as shown below (see Figs. 3 and 4(b)).

$$
\begin{aligned}
& d_{y, I-1 / 2}^{n+1 / 2}=d_{y, I-1 / 2}^{n-1 / 2}-\left(h_{I}^{n}-h_{I-1}^{n}\right)-g^{n-1 / 4}-g^{n+1 / 4}, \\
& e_{y, I-1 / 2}^{n+1 / 2}=(Z \Delta w) d_{y, I-1 / 2}^{n+1 / 2} /\left(3 / 2-\Delta l_{\mathrm{A}}\right)
\end{aligned}
$$

Then, $f^{n+3 / 4}$ and $g^{n+3 / 4}$ in domain (III) are (see Fig. 4(a))

$$
f^{n+3 / 4}=f^{n+1 / 4}+e_{I-1 / 2}^{n+1 / 2}, g^{n+3 / 4}=(\Delta w / 4 / Z) f^{n+3 / 4} .
$$

Then, $d$ and $e$ in connecting domain (III) are updated as (see Figs. 3 and 4(b))

$$
\begin{aligned}
& d_{y, I+1 / 2}^{n+3 / 4}=d_{y, I+1 / 2}^{n+1 / 4}-h_{I+1}^{n+1 / 2}+g^{n+1 / 4}+g^{n+3 / 4}, \\
& e_{y, I+1 / 2}^{n+3 / 4}=(Z \Delta w / 2) d_{y, I+1 / 2}^{n+3 / 4} /\left(1 / 2+\Delta l_{\mathrm{A}}+\Delta w^{2} / 8\right)
\end{aligned}
$$

In domain (I), $b$ and $h$ are updated as

$$
\begin{aligned}
& b_{i}^{n+1}=b_{i}^{n}-\left(e_{y, i+1 / 2}^{n+1 / 2}-e_{y, i-1 / 2}^{n+1 / 2}\right), \\
& h_{i}^{n+1}=(\Delta w / Z) b_{i}^{n+1} \quad(i \leq I-1) .
\end{aligned}
$$

In domain (II), $b$ and $h$ are updated as

$$
\begin{aligned}
b_{i}^{n+1} & =b_{i}^{n+1 / 2}-\left(e_{y, i+1 / 2}^{n+3 / 4}-e_{y, i-1 / 2}^{n+3 / 4}\right), \\
h_{i}^{n+1} & =(\Delta w / 2 / Z) b_{i}^{n+1} \quad(i \geq I+1) .
\end{aligned}
$$




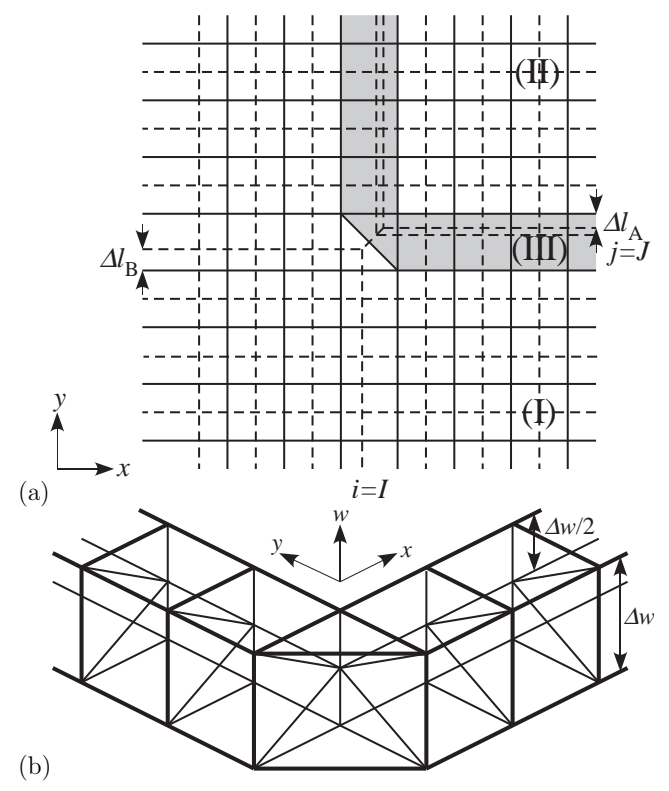

Fig. 5. Space-time grid: (a) spatial grid, and (b) domain (III).

In domain (III), $b$ and $h$ are updated as (see Fig. 4(a))

$$
b_{I}^{n+1}=-e_{y, I+1 / 2}^{n+3 / 4}+f^{n+3 / 4}, h_{I}^{n+1}=(\Delta w / 2 / Z) b_{I}^{n+1} .
$$

\section{3D Space-Time Grid with 2D Space}

The explicit time-marching scheme presented in the preceding subsection can be extended to that for a 3D space-time grid with $2 \mathrm{D}$ space. Fig. 5 presents a $3 \mathrm{D}$ space-time grid that has three domains (I), (II), and (III), similarly to the 2D space-time grid with 1D-space. Fig. 6 portrays electromagnetic variables at the corner of the joint part. The variable relations at the corner part are given as follows.

From Fig. 6(b), $f_{I, J}^{n+1 / 4}$ and $g_{I, J}^{n+1 / 4}$ are given as

$$
\begin{aligned}
& f_{I, J}^{n+1 / 4}=e_{x I, J+1 / 2}^{n+1 / 4}-e_{y I+1 / 2, J}^{n+1 / 4}+f_{I, J}^{n}, \\
& g_{I, J}^{n+1 / 4}=(\Delta w / 2 / Z) f_{I, J}^{n+1 / 4} .
\end{aligned}
$$

From Figs. 6(c) and 6(d), $d_{x I, J+1 / 2}^{n+1 / 2}, d_{y I+1 / 2, J}^{n+1 / 2}, d_{I, J}^{n+1 / 2}$ and $e_{I, J}^{n+1 / 2}$ are given as

$$
\begin{aligned}
d_{x I, J+1 / 2}^{n+1 / 2} & =d_{x I, J+1 / 2}^{n+1 / 4}+\left(g_{I, J+1}^{n+1 / 4}-g_{I, J}^{n+1 / 4}\right) \\
d_{y I+1 / 2, J}^{n+1 / 2} & =d_{y I+1 / 2, J}^{n+1 / 4}-\left(g_{I+1, J}^{n+1 / 4}-g_{I, J}^{n+1 / 4}\right) \\
d_{I, J}^{n+1 / 2} & =d_{I, J}^{n-1 / 2}-h_{I, J}^{n}+g_{I, J}^{n-1 / 4}+g_{I, J}^{n}+g_{I, J}^{n+1 / 4}, \\
e_{I, J}^{n+1 / 2} & =(Z \Delta w) d_{I, J}^{n+1 / 2} /\left(1-\Delta l_{\mathrm{A}}-\Delta l_{\mathrm{B}}\right)
\end{aligned}
$$

From Figs. 6(b) and 6(d), $f_{I, J}^{n+3 / 4}$ and $g_{I, J}^{n+3 / 4}$ are given as

$$
\begin{aligned}
& f_{I, J}^{n+3 / 4}=f_{I, J}^{n+1 / 4}+e_{x I, J+1 / 2}^{n+1 / 2}-e_{y I+1 / 2, J}^{n+1 / 2}-e_{I, J}^{n+1 / 2}, \\
& g_{I, J}^{n+3 / 4}=(\Delta w / 2 / Z) f_{I, J}^{n+3 / 4} .
\end{aligned}
$$

From Fig. 6(c), $d_{x I, J+1 / 2}^{n+3 / 4}$ and $d_{y I+1 / 2, J}^{n+3 / 4}$ are

$$
\begin{aligned}
& d_{x I, J+1 / 2}^{n+3 / 4}=d_{x I, J+1 / 2}^{n+1 / 2}+g_{I, J+1}^{n+3 / 4}-g_{I, J}^{n+3 / 4} \\
& d_{y I+1 / 2, J}^{n+3 / 4}=d_{y I+1 / 2, J}^{n+1 / 2}-g_{I+1, J}^{n+3 / 4}+g_{I, J}^{n+3 / 4} .
\end{aligned}
$$



Fig. 6. Electromagnetic variables at the corner of the joint part: (a) two prism elements, (b) electromagnetic variables for the primal grid, (c) electromagnetic variables for the sub grid, and (d) directions of $e$ and $d$.

From Fig. 6(b), $f_{I, J}^{n+1}$ and $g_{I, J}^{n+1}$ are

$$
\begin{aligned}
& f_{I, J}^{n+1}=-e_{y I+1 / 2, J}^{n+3 / 4}+e_{x I, J+1 / 2}^{n+3 / 4}+f_{I, J}^{n+3 / 4}, \\
& g_{I, J}^{n+1}=(\Delta w / Z) f_{I, J}^{n+1} .
\end{aligned}
$$

From Figs. 6(b) and 6(d), $b_{I, J}^{n+1}$ and $h_{I, J}^{n+1}$ are

$$
\begin{aligned}
b_{I, J}^{n+1} & =b_{I, J}^{n}-e_{x I, J-1 / 2}^{n+1 / 2}+e_{y I-1 / 2, J}^{n+1 / 2}+e_{I, J}^{n+1 / 2}, \\
h_{I, J}^{n+1} & =(2 \Delta w / Z) b_{I, J}^{n+1} .
\end{aligned}
$$

When $\varepsilon$ and $\mu$ are nonuniform, it is convenient to use variable $t$ instead of $w$ changing the metric dependently on $\varepsilon$ and $\mu$. The extension of the method above to a 4D space-time FI method is possible, but it is not straightforward because analogical discussion based on (6) and (7) is difficult to apply to $4 \mathrm{D}$ space-time.

\section{Application to Wave Computation}

Wave propagation induced by a magnetic current source is analyzed to examine the space-time FI method. For simplicity, the permittivity and permeability are set uniformly to unity by normalization. The magnetic current source with normalized frequency of 0.1 is located as depicted in Fig. 7(a). Fig. 7(b) depicts a space-time primal grid with $2 \mathrm{D}$-space. Domains (I) and (II) have a uniform time-step $\Delta w$ and $\Delta w / 2$, respectively, which are connected by the domain (III). The FI method can use prism elements in domain (II) similarly to the usual brick 

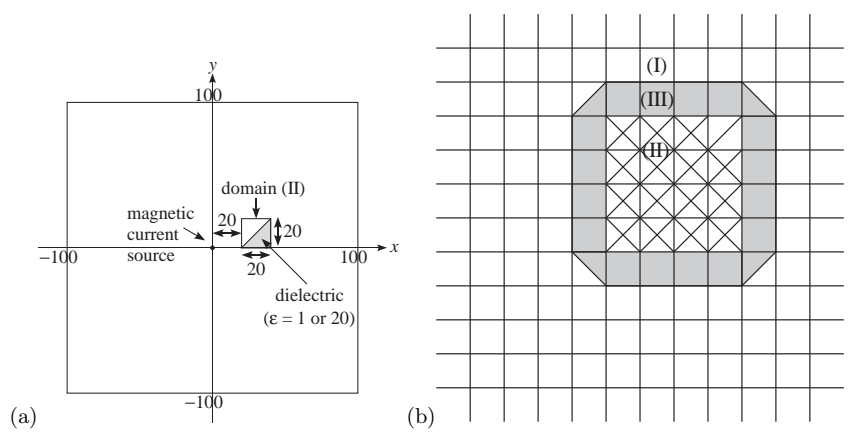

Fig. 7. Geometry of application example and space-time grid: (a) source and domain (II), and (b) three domains where the number of elements does not correspond to the simulations shown in Figs. 8 and 9.
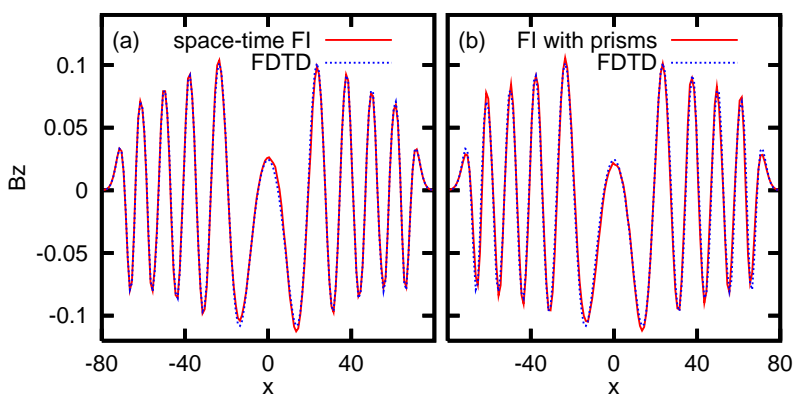

Fig. 8. Distributions of $B_{z}$ without scattering: (a) space-time FI method and (b) FI method with prism elements and uniform time-step.

elements. The spatial cell size is unity and $\Delta w=0.5$ in domain (I). The space-time FI method yields a distribution of $B_{z}$ as portrayed in Fig. 8(a) at $w=80$ and $y=10$, which agrees with that given by the conventional FDTD method having the same spatial grid and time-step as in domain (I). For comparison, Fig. 8(b) shows the distribution obtained from the FI method with uniform time-step using similar prism elements partially. Small discrepancies in the distributions are seen in Figs. 8(a) and (b), which are caused by nonuniform computational grids. The discrepancy can be reduced by the use of sufficiently small spatial grid size compared with the wavelength.

Next, wave scattering by a triangle dielectric is analyzed. The dielectric has $\varepsilon=20$ and $\mu=1$, which occupies half of domain (II), as depicted in Fig. 7(a). Figs. 9(a) and (b) portray distributions of $B_{z}$ obtained from the space-time FI method and the FDTD method. The FDTD method results in insufficient resolution in the dielectric because of the fixed cell size. The space-time FI method can zoom in and out according to the locally required resolution of the electromagnetic wave. The simulation presented above for the space-time FI method requires about twice as much computation time as the FDTD method because of the fine space-time grid in domain (II). The FDTD method with half-sized spatial and time steps yields a similar distribution shown in Fig. 9(c) to that in Fig. 9(a), at the expense of about four times as much computation time as the space-time FI method.

\section{CONCLUSION}

A space-time FI method was applied to electromagnetic field computation for flexible time discretization depending
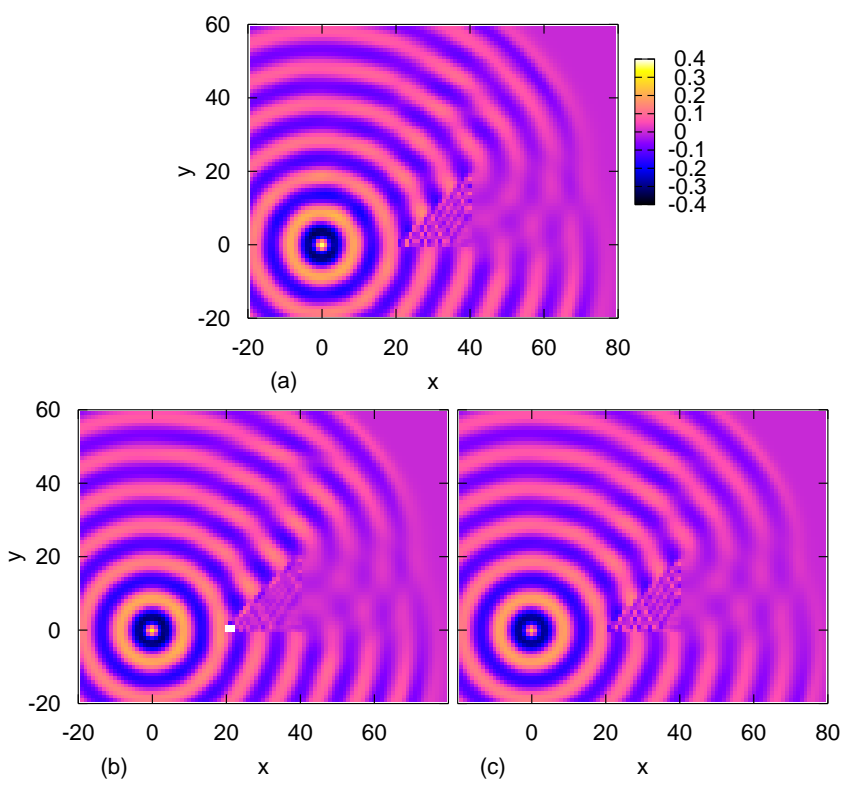

Fig. 9. Distributions of $B_{z}$ with scattering: (a) space-time FI method, (b) FDTD method, and (c) FDTD method with fine gird.

on nonuniform spatial discretization. Orthogonality between primal and sub grids was given by the Lorentz metric to obtain a constitutive equation for electromagnetic variables. An explicit time-marching scheme was presented.

Future work remains: discussion of the dispersion property of the space-time FI method, and extension of the proposed method to a $4 \mathrm{D}$ space-time FI method.

\section{ACKNOWLEDGMENT}

The author would like to express his gratitude to Mr. A. Kameari, Science Solutions International Laboratory, Inc. for his advice on the orthogonality in space-time.

\section{REFERENCES}

[1] K. S. Yee, "Numerical solution of initial boundary value problems involving Maxwellfs equations in isotropic media," IEEE Trans. Antennas Propagat., vol. 14, pp. 302-307, May 1966.

[2] T. Weiland, "Time domain electromagnetic field computation with finite difference methods," Int. J. Numer. Model., vol. 9, pp. 295-319, 1996.

[3] I. E. Lager, E. Tonti, A.T. de Hoop, G. Mur, and M. Marrone, "Finite formulation and domain-integrated field relations in electromagnetics - a synthesis," IEEE Trans. Magn., vol. 39, pp. 1199-1202, May 2003.

[4] M. W. Chevalier, R.J. Luebbers, and V.P. Cable, "FDTD local grid with material traverse," IEEE Trans. Antennas Propagat., vol. 45, pp. 411-412, March 1997.

[5] P. Thoma and T. Weiland, "A consistent subgridding scheme for the finite difference time domain method," Int. J. Numer. Model., vol. 9, pp. 359374, 1996.

[6] A.J. Butler and Z.J. Cendes, "Space-time finite elements derived by convolution for the efficient solution of transient eddy current problems," IEEE Trans. Magn., vol. 24, pp. 2688-2690, Nov. 1988.

[7] T. Renyuan, L. Feng, L. Yan and C. Xiang, "Analysis of transient nonlinear eddy current fields by space-time finite element method," IEEE Trans. Magn., vol. 34, pp. 2577-2580, Sept. 1998.

[8] S. Gyimóthy, A. Vágvölgyi, and I. Sebestyén, "Application of optimally distorted finite elements for field calculation problems of electromagnetism," IEEE Trans. Magn., vol. 38, pp. 365-368, March 2002. 